\title{
MODELAGEM DAS VARIÁVEIS DO ENSAIO DE DESGASTE DO REVESTIMENTO DE CARBONETO DE CROMO
}

\author{
MODELING THE CHROMIUM CARBIDE COATING WEAR TEST VARIABLES
}

\author{
Manoel Henrique Alves \\ Annelise Kopp Alves ${ }^{2}$
}

\begin{abstract}
RESUMO: Este trabalho tem como objetivo avaliar numericamente a taxa média de desgaste do revestimento de carboneto de cromo na matriz $\mathrm{NiCr}$ aspergido via $\mathrm{HVOF}$ em aço, através da regressão multivariada. A modelagem é realizada através de variáveis importantes de entrada no mecanismo de desgaste, a carga estática, velocidade e distância de deslizamento. Para isso foi feito uma pesquisa em artigos publicados em revistas internacionais para formar um banco de dados sobre esse revestimento, com dados experimentais já realizados. Com isso é modelado uma previsão da taxa média de desgaste do revestimento de acordo com tais variáveis gerando uma equação previsora. A modelagem se mostrou forte numericamente para prever o quanto o revestimento vai se desgastar. Pode-se verificar que a carga estática é uma variável determinante na previsão da taxa média de desgaste, sendo uma grandeza diretamente proporcional.
\end{abstract}

Palavras-Chaves: Desgaste. Revestimento. HVOF. Regressão.

ABSTRACT: This work aims to numerically evaluate the average wear rate of the chromium carbide coating in the $\mathrm{NiCr}$ matrix sprayed via $\mathrm{HVOF}$ on steel, through multivariate regression. The modeling is performed through important input variables in the wear mechanism, the static load, speed and sliding distance. For this, a search was made on articles published in international magazines to form a database on this coating, with experimental data already carried out. With this, a forecast of the average wear rate of the coating is modeled according to these variables, generating a predictive equation. The modeling proved to be numerically strong to predict how much the coating will wear out. It can be seen that the static load is a determining variable in the prediction of the average wear rate, being a directly proportional quantity.

Keywords: Wear. Coating. HVOF.Regression.

\section{INTRODUÇÃO}

A aspersão térmica surgiu com o objetivo de agregar resistência ao desgaste e à corrosão para o substrato, auxiliando na diminuição de custo e otimização dos processos. E com ela, seus diferentes processos de implementação, como o de HVOF (High Velocity Oxy-Fuel Flame), que é o processo de fabricação do objeto de estudo deste trabalho. Uma das aplicações de interesse é no revestimento de

\footnotetext{
${ }^{1}$ Mestrando em Engenharia na UFRGS, professor na UNIRITTER e servidor federal técnico da UFRGS. Email:manoel.henrique@ufrgs.br.

${ }^{2}$ Doutora em Engenharia pela UFRGS, professora associada na Escola de Engenharia da UFRGS. E-mail: annelise.alves@ufrgs.br.
} 
tubos de caldeiraria, que são amplamente utilizadas nas indústrias de mineração, gás e petróleo, de transformação entre tantos outros, ou como também nas aplicações em eixos de bombas, afim de reduzir a taxa de desgaste.

O carboneto de cromo é um dos materiais depositados via HVOF, sendo amplamente utilizado como forma de revestimento protetor, o qual promove alta resistência a corrosão e ao desgaste, inclusive em altas temperaturas.

O principal problema de pesquisa é a avaliação da taxa de desgaste do revestimento de carboneto de cromo na matriz $\mathrm{NiCr}$ em função das variáveis do ensaio de desgaste. Tal problema busca como hipótese de resultado, uma modelagem das variáveis de entrada e de saída como forma de compreensão do fenômeno.

Para obtenção dessa modelagem utiliza-se o método estatístico de regressão multivariada, selecionando as variáveis de entrada que fisicamente interferem na variável de saída. O conjunto de dados selecionados para formar o database deve representar a mais próxima realidade do problema.

Como objetivo geral desta pesquisa, busca-se compreender através do método de regressão multivariada a variação da taxa de desgaste do revestimento de carboneto de cromo aspergido por HVOF em função de suas variáveis.

Como objetivos específicos tem-se:

i. Elaborar modelo matemático considerando como resposta a taxa de desgaste e como variáveis: carga estática, velocidade de deslizamento e distância de deslizamento;

ii. Analisar a influência e significância das variáveis na taxa média de desgaste do revestimento;

iii. Verificar quais são as variáveis predominantes para estimar a taxa média de desgaste.

\section{METODOLOGIA}

Para formação de um banco de dados aplicável para as respostas esperadas, foi necessário selecionar os artigos que traziam informações que auxiliam a modelagem e interpretação do comportamento do sistema. Para este trabalho, foi realizada uma pesquisa de artigos publicados no período de 2016 a 2021, em revistas internacionais e que utilizaram metodologia experimental acerca do revestimento de carboneto de cromo na matriz ${ }_{25} \mathrm{NiCr}$, e que apresentam dados completos acerca do ensaio de desgaste. Os artigos utilizados são os listados nas referências bibliográficas.

O principal objetivo desse método é equacionar um modelo que explique numericamente a relação de uma variável de resposta com uma ou mais variáveis controladoras de entrada do problema. 
De maneira analítica, o método com os dados amostrais passa por uma aritmética matricial para obtenção dos coeficientes betas. Consistindo em:

$$
\begin{aligned}
& \beta=\left(x^{T} \cdot x\right)^{-1} \cdot x^{T} \cdot y \\
& \beta=\left(x^{T} \cdot x\right)^{-1} \cdot x^{T} \cdot y
\end{aligned}
$$

$\beta=\left(x^{T} \cdot x\right)^{-1} \cdot x^{T} \cdot y$

Evidenciando uma predição da regressão por meio dos valores desses coeficientes. Com isso, precisa-se compreender a variação dos dados e ajustá-los em relação às saídas. Para isso é adotado uma análise de variância consolidada para esse método, conhecida como ANOVA (Figura I), e disponível no software Excel, que se trata de uma forma de conferir se os efeitos são numericamente significativos ou não (Piovesan, 2016).

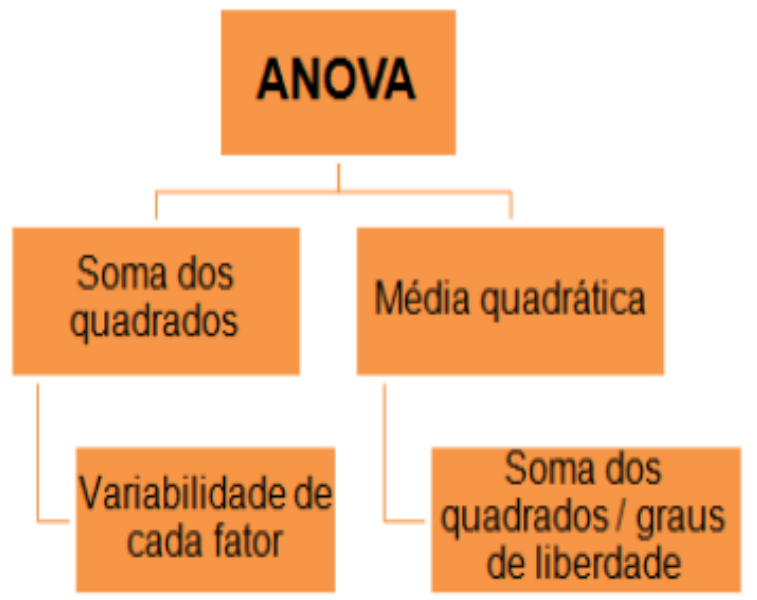

Figura I - Passos para análise de variância ANOVA (Fonte: Piovesan, 2016).

Um dos métodos de estimação mais aplicados nesse modelo e com melhor precisão é o dos mínimos quadrados que define a equação mais próxima dos dados experimentados. De acordo com as relações tabeladas, ou seja, para cada " $x$ " entrada implicando em uma variável de saída, esse método de estimação define os coeficientes de maneira que a soma dos quadrados dos erros seja a menor possível da seguinte maneira:

$$
\text { Soma dos quadrados }=\sum_{i=1}^{n} \varepsilon_{i}^{2}=\sum_{1-1}^{n}\left(Y_{i}-a-b . X_{i}\right)
$$

Onde $\varepsilon$ é o erro, o distanciamento do valor real, e a e b são fatores estimadores, sendo: 


$$
b=\frac{\sum x_{i} y_{i}-n y x}{\sum x_{i}^{2}-n x^{2}} \quad \text { e } \quad a=y-b x
$$

Com isso, é possível obter a equação de regressão múltipla do problema modelado. Ela se apresenta na seguinte forma:

$$
Y=\alpha+\beta_{1} x_{1}+\beta_{2} x_{2}+\beta_{3} x_{3} \ldots+\varepsilon
$$

Onde as variáveis $\mathrm{x}$ são as de entrada, enquanto a $Y$ é a resposta do problema. Os coeficientes $\alpha$ e $\beta$ são valores obtidos pelo método estatístico, enquanto $\varepsilon$ é o erro previsto na modelagem. $O$ ajuste do modelo é feito por meio de $\mathrm{R}^{2}$, p-valor e ANOVA.

Após ajuste do modelo com a análise da variância, é necessário realizar a análise dos resíduos (diferença entre a variável de resposta estimada com a variável de resposta observada). Com a análise de resíduos espera-se verificar se o modelo utilizado é adequado para os dados observador. Existem também algumas formas de se avaliar os resíduos, chamados de pressupostos, e para essa modelagem será utilizado o pressuposto de variância constante (por ser uma regressão multivariada), é o diagnóstico da homocedasticidade.

O diagnóstico da homocedasticidade é simples e gráfico, é plotado um gráfico dos resíduos padrões gerados para cada observação de taxa de desgaste (resposta). Assim espera-se que os pontos de resíduos sejam aleatórios, sem nenhuma tendência na forma de funil ou linear (crescente ou decrescente).

Equacionando assim uma equação que possua como resposta $\mathrm{Y}$ a taxa média de desgaste $\left(\mathrm{mm}^{3} / \mathrm{mN}\right)$, em função das seguintes variáveis:

- Xi é a carga estática em N;

- $\mathrm{X}_{2}$ é velocidade de deslizamento $\mathrm{em} \mathrm{m} / \mathrm{s}$

- $\mathrm{X}_{3}$ é a distância de deslizamento em $\mathrm{m}$.

\section{RESULTADOS E DISCUSSÃO}

Para essa análise foram escolhidas variáveis que são características específicas do ensaio de desgaste abrasivo, a velocidade de deslizamento, a carga estática e a distância percorrida, são variáveis completamente independentes, mas que se relacionam diretamente com a taxa média de desgaste, essencial para aplicação desse método.

Assim com base no banco de dados, foram selecionados os resultados que contemplem todas as variáveis de interesse dessa análise, assim obteve-se 29 observações possíveis de serem utilizadas como entrada, e efetuando o tratamento de dados da modelagem, o refinamento de qualidade, obtevese 20 observações (Tabela I). 
Tabela I - Dados de entrada

\begin{tabular}{|c|c|c|c|}
\hline $\mathrm{X} 1$ & $x_{2}$ & X3 & $\mathrm{Y}$ \\
\hline $\begin{array}{l}\text { Carga } \\
\text { estática } \\
\text { (N) }\end{array}$ & $\begin{array}{l}\text { velocidade de } \\
\text { deslizamento } \\
(\mathrm{m} / \mathrm{s})\end{array}$ & $\begin{array}{l}\text { distância de } \\
\text { deslizamento } \\
\text { (m) }\end{array}$ & $\begin{array}{l}\text { Taxa média de desgaste } \\
\qquad\left(\mathrm{mm}^{3} / \mathrm{mN}\right)\end{array}$ \\
\hline 10 & 0,6 & 750 & $3,20 \mathrm{E}-04$ \\
\hline 20 & 0,6 & 750 & $5,00 \mathrm{E}-04$ \\
\hline 30 & 0,6 & 750 & $9,50 \mathrm{E}-04$ \\
\hline 10 & 0,1 & 5000 & $1,58 \mathrm{E}-06$ \\
\hline 10 & 0,1 & 5000 & $3,13 \mathrm{E}-06$ \\
\hline 15 & 0,25 & 1256 & $5,20 \mathrm{E}-04$ \\
\hline 15 & 0,5 & 1256 & $4,80 \mathrm{E}-04$ \\
\hline 15 & 1 & 1256 & $3,30 \mathrm{E}-04$ \\
\hline 15 & 1,5 & 1256 & $2,50 \mathrm{E}-04$ \\
\hline 30 & 0,25 & 1256 & $7,50 \mathrm{E}-04$ \\
\hline 30 & 0,5 & 1256 & $6,50 \mathrm{E}-04$ \\
\hline 30 & 1 & 1256 & $4,00 \mathrm{E}-04$ \\
\hline 45 & 0,25 & 1256 & $1,05 \mathrm{E}-03$ \\
\hline 45 & 0.5 & 1256 & $8,50 \mathrm{E}-04$ \\
\hline 60 & 0,25 & 1256 & $1,45 E-03$ \\
\hline 60 & 0,5 & 1256 & $1,30 \mathrm{E}-03$ \\
\hline 5 & 0,1 & 3000 & $5,00 \mathrm{E}-12$ \\
\hline 5 & 0,2 & 3000 & $2,80 \mathrm{E}-11$ \\
\hline 10 & 0,1 & 5000 & $2,00 \mathrm{E}-06$ \\
\hline 10 & 0,1 & 5000 & $9,00 \mathrm{E}-05$ \\
\hline
\end{tabular}

Assim efetuando a regressão multivariada, primariamente pode-se obter a estatística da regressão, compilada na Tabela 2.

Tabela 2 - Estatística de regressão

\begin{tabular}{|l|r|}
\hline \multicolumn{2}{|c|}{ Estatística de regressão } \\
\hline R múltiplo & 0,983219 \\
\hline R-Quadrado & 0,96672 \\
\hline Erro padrão & 0,00013 \\
\hline Observações & 20 \\
\hline
\end{tabular}

Pode-se visualizar que o $\mathrm{R}$ múltiplo está elevado, também expressando uma boa correlação entre as variáveis com vistas a resposta do modelo.

Nessa modelagem o R-quadrado está indicando que $96,67 \%$ dos dados de previsão coincidem com os dados do banco de dados, como pode-se ver na curva de previsão de alguma das variáveis, como ilustram as Figuras 2 e 3. O R-quadrado expõe a qualidade do modelo em relação às demais modelagens, visto a abrangência eficaz da cobertura dos dados de entrada. Para uma regressão multivariada é fundamental que o modelo apresenta o $\mathrm{R}^{2}$ elevado, é o chamado teste de colinearidade 
ou multicolinearidade da regressão. Essa porcentagem não considera o desvio do erro padrão, que indica a possibilidade de variação para mais ou para menos.

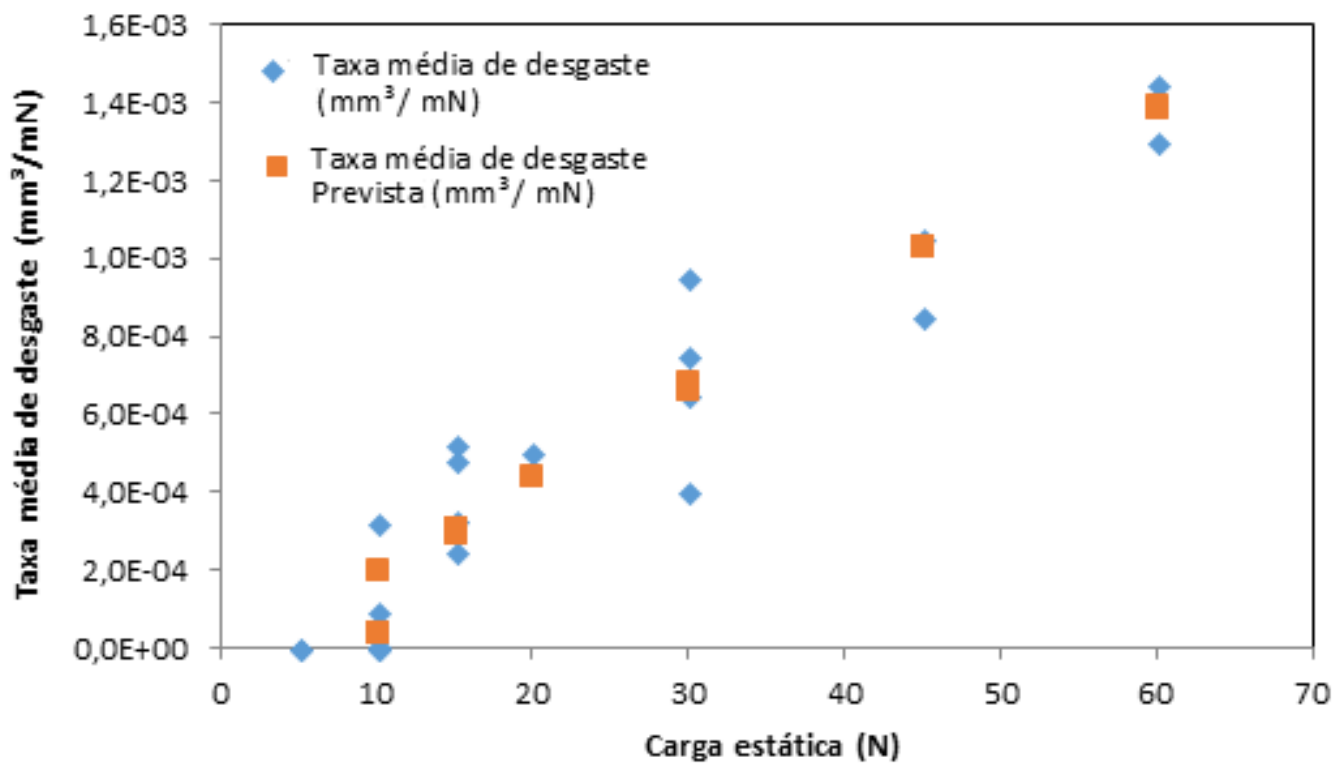

Figura 2I - Previsão da taxa de desgaste em relação à carga estática

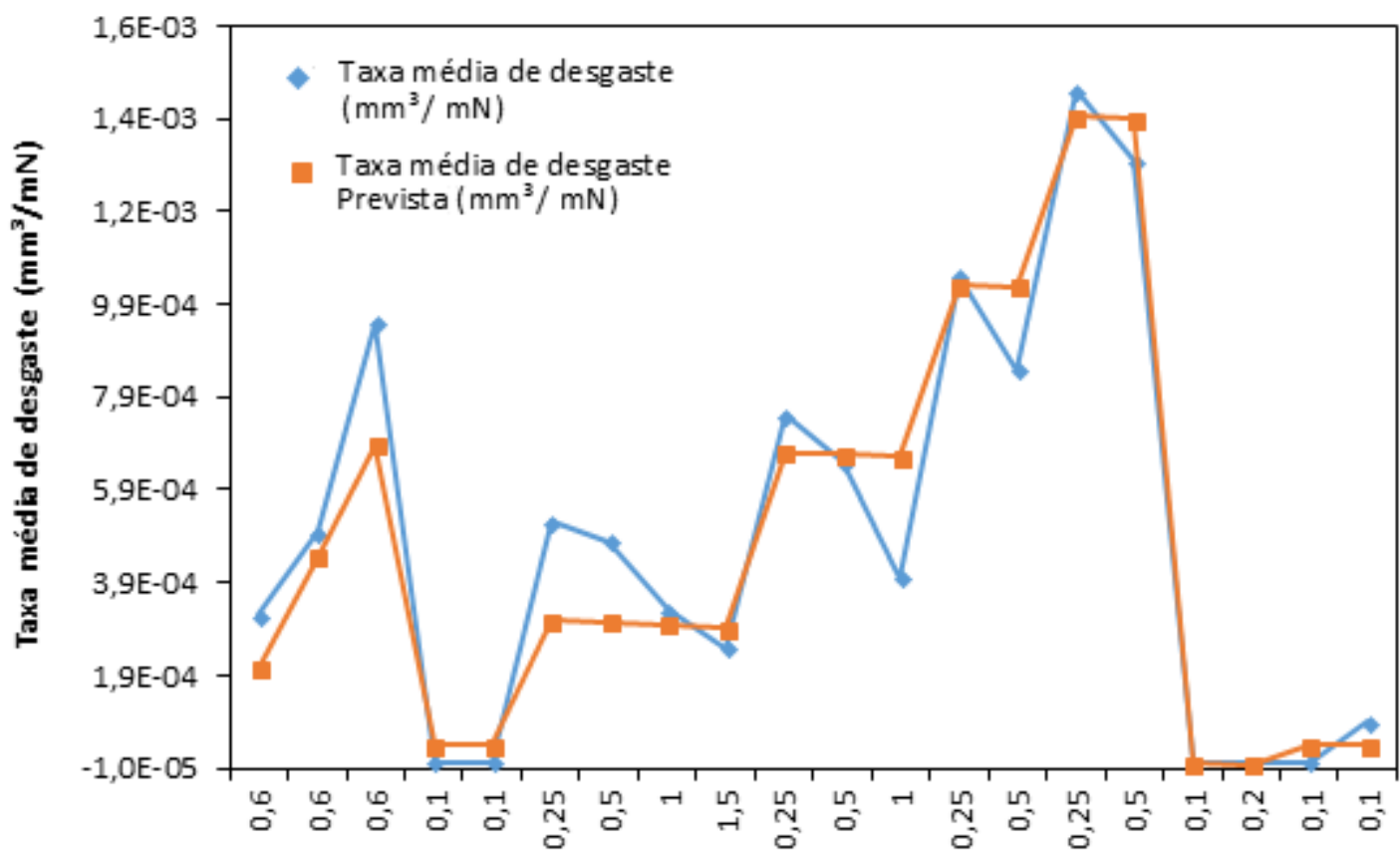

Velocidade de deslizamento $(\mathrm{m} / \mathrm{s})$

Figura 3 - Previsão da taxa de desgaste em relação a velocidade de deslizamento

$\mathrm{Na}$ Figura 2 pode-se visualizar, mesmo sendo um gráfico de análise de $\mathrm{R}^{2}$, que existe uma tendência crescente em relação à carga estática e o desgaste. De acordo com a pesquisa de Bolelli et 
al. (2016), que avaliou o comportamento tribológico do mesmo revestimento de carboneto de cromo, constatou que conforme se aumenta a carga estática sobre a amostra, há aumento na perda de massa do revestimento através do mecanismo de desgaste abrasivo.

Matikainen et al. (2017) também mostraram em sua pesquisa a comparação da perda de massa de aço carbono, quando há aumento da carga estática, com a finalidade de comparar a redução do desgaste quando o substrato é revestido, verificando que uma amostra estando revestida ou não apresenta maior taxa de desgaste quando há aumento da carga estática, contudo o revestimento reduziu em mais de $99 \%$ a taxa de desgaste. Neste trabalho, o aumento da taxa média de desgaste com a carga estática é constatado nesta modelagem através de uma relação linear.

Esse efeito é esperado também porque quanto maior é a carga estática, maior é o coeficiente de atrito que causa remoção por fricção na superfície, e consequentemente desprende material da estrutura do revestimento, aumentando a taxa média de desgaste. Esse fenômeno também é encontrado nos trabalhos de Vashishtha e Sapate (2017) e de Vashishtha et al. (2018).

Nessa modelagem embora não tenha sido analisado o coeficiente de atrito, a carga estática, a velocidade de deslizamento e a distância de deslizamento são variáveis nas quais o atrito é dependente delas, ou seja, ele é intrínseco à análise, e pode-se avaliar que ele está presente nos coeficientes da Tabela 3.

Karaoglanli et al. (2017) expõem uma equação que relaciona a taxa média de desgaste, e as variáveis utilizadas nessa modelagem. A pesquisa valida os dados experimentais por meio da seguinte equação:

$$
K=\frac{V}{w \cdot S}
$$

Onde $\mathrm{K}$ é a taxa média de desgaste, $\mathrm{V}$ é o volume desgastado, w é a carga nominal do ensaio e s é a distância de deslizamento. Com isso percebe-se que esta modelagem 3 condiz com a significância das variáveis para o resultado de previsão da taxa de desgaste, condizendo fisicamente com o esperado.

Karaoglanli et al. (2017) também explicam que nesse equacionamento conhecer a velocidade de deslizamento é fundamental, pois os experimentos comprovaram que a velocidade deslizamento elevam o volume desgastado, e sua influência é ainda maior para altas velocidades, além de também comprovarem que a taxa de desgaste aumenta quando a carga nominal é aumentada, validando também o gráfico de previsão da Figura 14.

A Figura 3 mostra a previsão da taxa média de desgaste com a velocidade de deslizamento. Segundo Vashishtha e Sapate (2017) em sua pesquisa acerca do mapeamento de desgaste abrasivo do revestimento de carboneto de cromo via HVOF, não existe uma relação simples entre coeficiente de 
atrito e o desgaste. A pesquisa mostrou que conforme a velocidade de deslizamento aumenta, a taxa de desgaste diminui devido a mudança do coeficiente de atrito. Contudo, para velocidade muito baixas, o coeficiente de atrito é alto, o que aumenta a taxa média de desgaste.

Ainda sobre a Figura 3, a principal intenção de se evidenciar os pontos de previsão sobre os pontos de resposta experimental, com esse tipo de gráfico, é poder visualizar que o modelo de previsão está próximo da resposta experimental do banco de dados, podendo assim confirmar a força numérica da modelagem para prever o comportamento de desgaste com as variáveis selecionadas, consolidando o valor de $\mathrm{R}^{2}$ obtido.

Por conseguinte, a principal informação que se espera desse método é a obtenção dos coeficientes que determinam a curva de previsão do modelo relacionando as variáveis. A Tabela 3 mostra os coeficientes obtidos, e pode-se visualizar o valor-P, que indica qual das variáveis são mais significativas e predominantes em determinar a taxa de desgaste. A velocidade de deslizamento desenvolvida durante o ensaio de desgaste mostrou-se numericamente mais determinante para se estimar o quanto o revestimento pode se desgastar. Vashishtha e Sapate (2017) variaram a velocidade de deslizamento para obtenção de um mapeamento de desgaste e constataram altas diferenças da taxa de desgaste com a variação da velocidade, sendo esta também uma variável crucial para compreensão desse mecanismo.

Tabela I - Resultados da metodologia de regressão

\begin{tabular}{|l|c|c|c|c|c|c|}
\hline & Coeficientes & $\begin{array}{c}\text { Erro } \\
\text { padrão }\end{array}$ & Stat t & valor-P & $\begin{array}{c}\mathbf{9 5 \%} \\
\text { inferiores }\end{array}$ & $\begin{array}{c}\mathbf{9 5 \%} \\
\text { superiores }\end{array}$ \\
\hline Carga estática & $2,41 \mathrm{E}-05$ & $1,42 \mathrm{E}-06$ & 17,0182 & $4,11 \mathrm{E}-12$ & $2,11 \mathrm{E}-05$ & $2,71 \mathrm{E}-05$ \\
\hline $\begin{array}{l}\text { Velocidade de } \\
\text { deslizamento }\end{array}$ & $-1,3 \mathrm{E}-05$ & $6,82 \mathrm{E}-05$ & $-0,19261$ & 0,849547 & $-0,00016$ & 0,000131 \\
\hline $\begin{array}{l}\text { Distância de } \\
\text { deslizamento }\end{array}$ & $-4 \mathrm{E}-08$ & $1,28 \mathrm{E}-08$ & $-3,1161$ & 0,006284 & $-6,7 \mathrm{E}-08$ & $-1,3 \mathrm{E}-08$ \\
\hline
\end{tabular}

A velocidade de deslizamento é um fator determinante na previsão da taxa de desgaste, assim como a distância de deslizamento. São fatores que se relacionam, mas são completamente independentes um do outro, por isso tão relevantes.

Mishra, Kumar e Sinha (2021) evidenciaram com sua pesquisa que existe uma faixa de velocidade de deslizamento crítica para o revestimento, ou seja, há uma velocidade na qual há um acentuamento no desgaste, pois é uma velocidade em que as partículas removidas se tornam um terceiro corpo no processo de desgaste, aumentando mais ainda essa taxa. Após se atingir esta faixa de velocidade há um decaimento e estabilidade na taxa de desgaste. Essa faixa crítica de velocidade é encontrada em baixas velocidades e como pode se observar na Figura 3, os pontos com maiores taxas de desgaste são localizados com $0,25 \mathrm{~m} / \mathrm{s}$. 
A Figura 4 pode-se avaliar a distribuição de probabilidade normal para os dados. Nesta distribuição percebe-se que há abrangência de dados, eles apresentam uniformidade e equidistância entre as amostras, fato que agrega a confiabilidade da previsão.

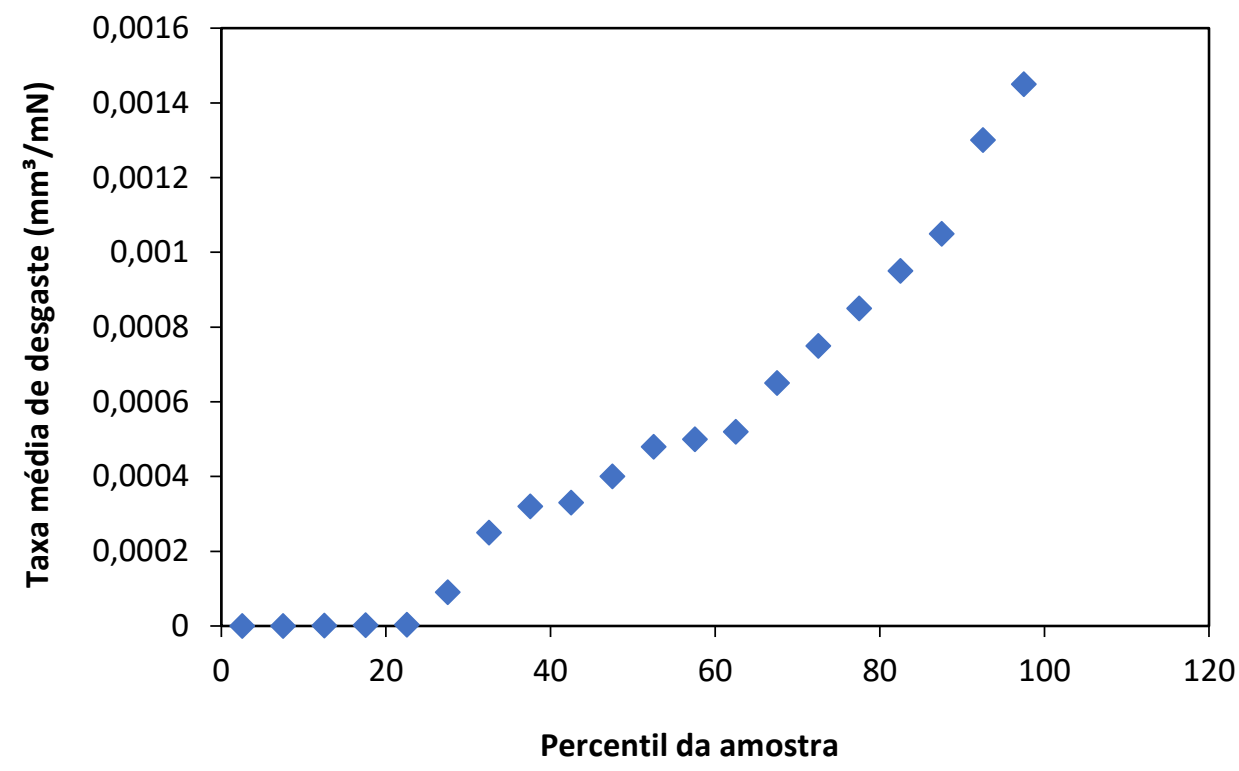

Figura 4- Gráfico de Distribuição normal

Para validação numérica do modelo foi feita a análise da variância da regressão, avaliando a adequação da modelagem através da ANOVA da regressão, exposta na Tabela 4.

Tabela 4 - Análise da Variância da Regressão

\begin{tabular}{|l|r|r|r|l|r|}
\hline & $\begin{array}{r}\text { Graus de } \\
\text { liberdade }\end{array}$ & $\begin{array}{r}\text { Graus de } \\
\text { liberdade }\end{array}$ & $\begin{array}{c}\text { Graus de } \\
\text { liberdade }\end{array}$ & $\boldsymbol{F}$ & $\begin{array}{c}\boldsymbol{F} \text { de } \\
\text { significação }\end{array}$ \\
\hline Regressão & 3 & $8,41 \mathrm{E}-06$ & $2,8 \mathrm{E}-06$ & 164,6034 & $3,1 \mathrm{E}-12$ \\
\hline Resíduo & 17 & $2,89 \mathrm{E}-07$ & $1,7 \mathrm{E}-08$ & & \\
\hline Total & 20 & $8,7 \mathrm{E}-06$ & & & \\
\hline
\end{tabular}

Como o valor $\mathrm{F}$ de significação é menor que o fator $\mathrm{F}$ de significação, pode-se avaliar que a regressão é significativa.

Desse modo utilizam-se os coeficientes da Tabela 4 como reguladores das variáveis da modelagem, sendo:

$$
\begin{aligned}
& \alpha=0 \\
& \beta_{1}=2,4 \mathrm{I} E-5 \\
& \beta_{2}=-1,3 \mathrm{E}-5 \\
& \beta_{3}=-4,0 \mathrm{E}-8
\end{aligned}
$$

Obtém-se, assim, a equação modeladora de regressão multivariada:

$$
Y\left(X_{1}, X_{2}, X_{3}\right)=2,4 I E-5 \cdot X_{1}-1,3 E-5 \cdot X_{2}-4, o E-8 \cdot X_{3}
$$


Onde $\mathrm{Y}$ é a taxa média de desgaste $\left(\mathrm{mm}^{3} / \mathrm{mN}\right)$, e as variáveis $\mathrm{X}_{\mathrm{I}}$ é a carga estática $(\mathrm{N}) ; \mathrm{X}_{2}$ é a velocidade de deslizamento $(\mathrm{m} / \mathrm{s})$ e; $\mathrm{X}_{3}$ é a distância de deslizamento $(\mathrm{m})$.

Em consonância com a metodologia proposta é realizada a análise de resíduos através do diagnóstico de homocedasticidade, que avalia se existe constância nos resíduos apresentado pela comparação dos dados de entrada com os dados de previsão. A Figura 5 a seguir mostra a distribuição dos resíduos padrões em função da previsão de resposta (taxa média de desgaste). Como não há nenhuma tendência (crescente ou decrescente) marcada graficamente, a variância é constante.

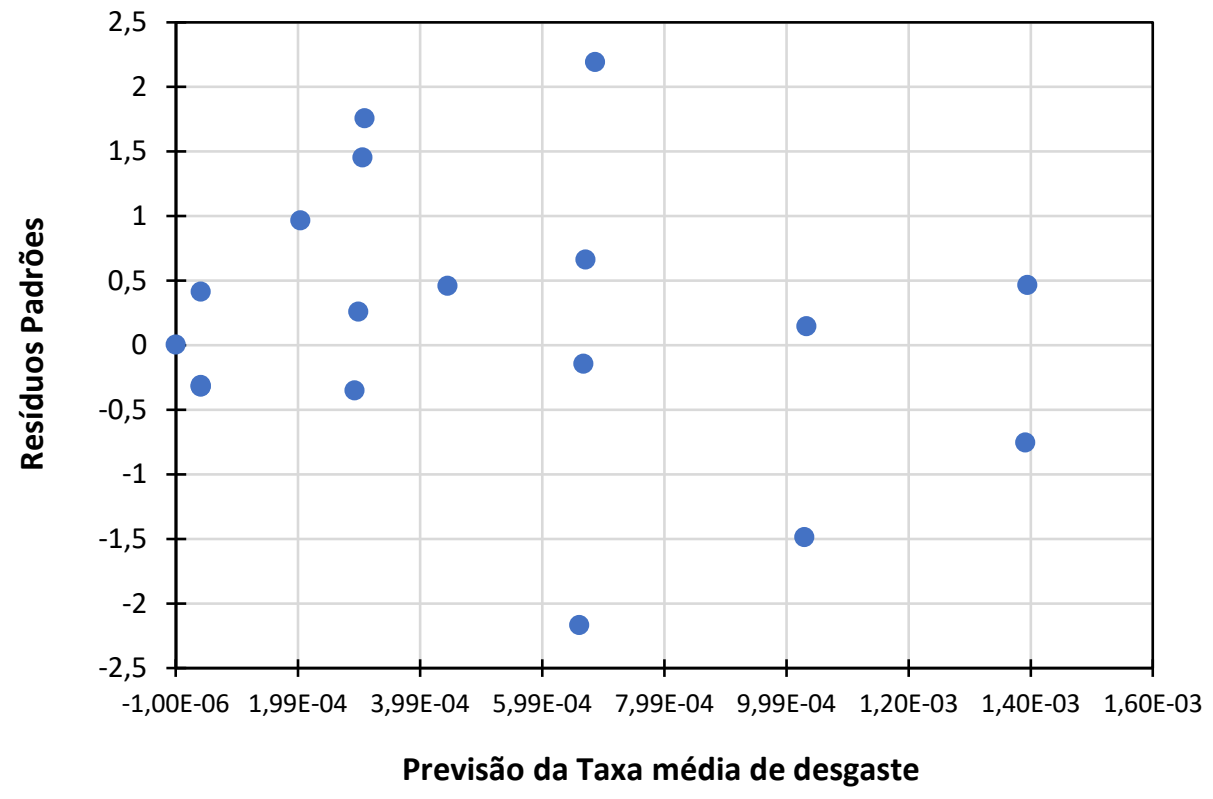

Figura 2 - Diagnóstico de homoscedasticidade da modelagem 3.

\section{${ }_{4}$ CONCLUSÕES}

Com base nos resultados numéricos encontrados através do método de regressão multivariada, pode-se mapear as principais variáveis no desgaste, com o objetivo de compreender a resistência ao desgaste do revestimento de carboneto de cromo na matriz $\mathrm{NiCr}$ aspergido via HVOF.

Com a modelagem foi possível equacionar $(\mathrm{Y})$ uma previsão da taxa média de desgaste $\left(\mathrm{mm}^{3} / \mathrm{mN}\right)$ considerando as principais variáveis e verificar sua predominância.

A modelagem previu como variável mais significativa dentre as analisadas a velocidade de deslizamento. Os valores de $\mathrm{R}^{2}$ e $\mathrm{R}$-múltiplo foram relevantes. Pode-se interpretar que estas propriedades se relacionaram com maior coerência na previsão da taxa média de desgaste. Nessa 
análise pode-se verificar uma tendência linear clara em relação a carga estática, sendo essa modelagem a mais forte para previsão no comportamento da taxa de desgaste.

Com base na coerência física e numérica exigidas nas modelagens, esta análise se mostrou satisfatória para realizar a previsão do comportamento da taxa de desgaste.

Assim os resultados numéricos obtidos, com a previsão da taxa de desgaste, podem ser usados como base para futuros testes experimentais, formando um planejamento experimental mais delineado e preciso.

\section{REFERÊNCIAS BIBLIOGRÁFICAS}

Bolelli,G.; Berger,L.M.; Börner,T.; Koivuluoto,H.; Matikainen,V.; Lusvarghi,L.; Lyphout,C.; Markocsan,N.; Nylén,P.; Sassatelli,P.; Trache,R.; Vuoristo,P. Sliding and abrasive wear behaviour of $\mathrm{HVOF}$ and HVAF sprayed $\mathrm{Cr}_{3} \mathrm{C}_{2}-\mathrm{NiCr}$ hardmetal coatings. Wear, V.359, 2016. In:〈https://doi.org/ı.1o16/j.wear.2016.03.034.>

Karaoglanli,A.C.; Oge,M.; Doleker,K.M.; Hotamis,M.; Comparison of tribological properties of HVOF sprayed coatings with different composition. Surface and Coatings Technology, V.318, 2017. In:〈https://doi.org/ro.1016/j.surfcoat.2017.02.021>

Matikainen,V.; Bolelli,G.; Koivuluoto,H.; Sassatelli,P.; Lusvarghi,L.; Vuoristo,P. Sliding wear behaviour of HVOF and HVAF sprayed $\mathrm{Cr}_{3} \mathrm{C}_{2}$-based coatings. Wear, V.389, 2017. In:〈https://doi.org/Io.1oI6/j.wear.2017.04.ooI>

Mishra,T.K.; Kumar,A.; Sinha,S.K. Experimental investigation and study of HVOF sprayed WC${ }_{12} \mathrm{Co}$, WC-roCo-4 $\mathrm{Cr}$ and $\mathrm{Cr}_{3} \mathrm{C}_{2-25} \mathrm{NiCr}$ coating on its sliding wear behaviour. International Journal of Refractory Metals and Hard Materials, V.94, 202I. In:〈https://doi.org/ı.1016/j.ijrmhm.2020.105404>

Piovesan, J. C. Otimização Multivariada Da Substituição De Agregados De Resíduos De Construção E Demolição Em Concreto. Dissertação UFAM-PPGEC. Manaus, 2016.

Vashishtha,N.; Sapate,S.G.; Sahariah,B. J.; Bagde,P. Microstructural characterization and wear behaviour of High Velocity Oxy-Fuel sprayed $\mathrm{Cr}_{3} \mathrm{C}_{2-25} \mathrm{NiCr}$ coating. Materials Today: Proceedings, V.5, Part 3, 2018. In<https://doi.org/ro.1016/j.matpr.2018.06.089>.

Vashishtha,N.; Sapate,S.G. Abrasive wear maps for High Velocity Oxy Fuel (HVOF) sprayed WC-12Co and $\mathrm{Cr}_{3} \mathrm{C}_{2}-25 \mathrm{NiCr}$ coatings. Tribology International, V.II4, 2017. In:〈https://doi.org/ro.1016/j.triboint.2017.04.037> 